\title{
Multispectral imaging of the ocular fundus using light emitting diode illumination
}

N. L. Everdell, I. B. Styles, A. Calcagni, J. Gibson, J. Hebden, and E. Claridge

Citation: Review of Scientific Instruments 81, 093706 (2010); doi: 10.1063/1.3478001

View online: https://doi.org/10.1063/1.3478001

View Table of Contents: http://aip.scitation.org/toc/rsi/81/9

Published by the American Institute of Physics

\section{Articles you may be interested in}

Versatile multispectral microscope based on light emitting diodes

Review of Scientific Instruments 82, 123106 (2011); 10.1063/1.3660810

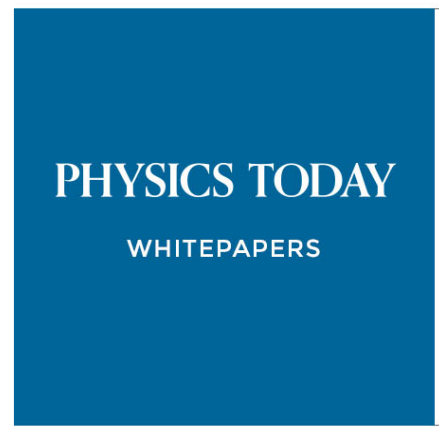

MANAGER'S GUIDE

Accelerate R\&D with

Multiphysics Simulation

\section{READ NOW}

PRESENTED BY $\checkmark \subset \bigcirc M S \bigcirc L$ 


\title{
Multispectral imaging of the ocular fundus using light emitting diode illumination
}

\author{
N. L. Everdell, ${ }^{1}$ I. B. Styles, ${ }^{2}$ A. Calcagni, ${ }^{2}$ J. Gibson, ${ }^{3}$ J. Hebden, ${ }^{1}$ and E. Claridge ${ }^{2}$ \\ ${ }^{1}$ Department of Medical Physics, Malet Place Engineering Building, University College London, \\ London WC1E 6BT, United Kingdom \\ ${ }^{2}$ School of Computer Science, University of Birmingham, Edgbaston, Birmingham B15 2TT, \\ United Kingdom \\ ${ }^{3}$ School of Life and Health Sciences, Aston University, Birmingham B4 7ET, United Kingdom
}

(Received 29 October 2009; accepted 19 July 2010; published online 29 September 2010)

\begin{abstract}
We present an imaging system based on light emitting diode (LED) illumination that produces multispectral optical images of the human ocular fundus. It uses a conventional fundus camera equipped with a high power LED light source and a highly sensitive electron-multiplying charge coupled device camera. It is able to take pictures at a series of wavelengths in rapid succession at short exposure times, thereby eliminating the image shift introduced by natural eye movements (saccades). In contrast with snapshot systems the images retain full spatial resolution. The system is not suitable for applications where the full spectral resolution is required as it uses discrete wavebands for illumination. This is not a problem in retinal imaging where the use of selected wavelengths is common. The modular nature of the light source allows new wavelengths to be introduced easily and at low cost. The use of wavelength-specific LEDs as a source is preferable to white light illumination and subsequent filtering of the remitted light as it minimizes the total light exposure of the subject. The system is controlled via a graphical user interface that enables flexible control of intensity, duration, and sequencing of sources in synchrony with the camera. Our initial experiments indicate that the system can acquire multispectral image sequences of the human retina at exposure times of $0.05 \mathrm{~s}$ in the range of $500-620 \mathrm{~nm}$ with mean signal to noise ratio of $17 \mathrm{~dB}$ (min 11, std 4.5), making it suitable for quantitative analysis with application to the diagnosis and screening of eye diseases such as diabetic retinopathy and age-related macular degeneration.

(c) 2010 American Institute of Physics. [doi:10.1063/1.3478001]
\end{abstract}

\section{INTRODUCTION}

It has long been recognized that imaging of the interior of the human eye (often referred to as the ocular fundus) is a valuable tool in the diagnosis and monitoring of a number of common medical conditions. Since the fundus allows direct, noninvasive visualization of blood vessels in vivo, it can yield valuable information on the general health of the subject. Fundus imaging is widely used to screen for diabetes, ${ }^{1}$ which is known to cause hemorrhages in the retinal blood vessels. Imaging of the fundus can also provide information about many eye-specific diseases such as age-related macular degeneration (AMD) (Ref. 2) and glaucoma, ${ }^{3}$ both of which are major causes of blindness.

A variety of imaging methods are used to examine the fundus. The most commonly used technique is conventional RGB color photography, and this is widely available in optometry clinics. This technique has the advantage of being quick and simple to perform in a primary care setting, as no contrast agents need to be administered to the patient. However, RGB photography acquires images from only three rather broad wavelength bands, and its ability to distinguish between different light absorbing biological molecules (chromophores) is very limited. This limitation prevents this technique from providing quantitative information that could be diagnostically useful.
The de facto imaging technique for examining blood vessels in the fundus is fluorescein angiography. A fluorescent contrast agent is injected into the bloodstream, and time-series images of the fundus taken at a specific wavelength are acquired to image the vasculature. This approach yields extremely high quality images of the vascular network of the fundus, but can only be performed under the supervision of a physician due to the potential for an adverse reaction to the contrast agent, and is therefore not suitable for use in primary care.

A recent development has been the introduction of the scanning laser ophthalmoscope (SLO), which is gradually becoming more popular in the clinical setting. A SLO acquires images by rapidly scanning a laser across the fundus, and recording the image one pixel at a time, typically obtaining excellent $(\sim 20 \mu \mathrm{m})$ spatial resolution from a very wide field of view, but the resulting images are often significantly spatially distorted.

Finally, optical coherence tomography (OCT) is now widely used to identify structural features in the fundus. ${ }^{4}$ OCT can provide information about retinal structures up to approximately $1 \mathrm{~mm}$ below the surface, but does not readily provide information about chromophore concentrations in the tissue.

There has been significant interest in recent years in the 
development of multispectral imaging techniques for the fundus. ${ }^{5-10}$ A multispectral image set $\mathrm{I}(x, y, \lambda)$, referred to as a spectral cube or a lambda stack, contains spatial (pixel) information along two dimensions $(x, y)$ and spectral information along the third dimension $\lambda$. With spectral data available at each image point $\mathbf{x}=(x, y)$ it is possible to derive quantitative maps of diagnostically important physiological parameters such as, for example, blood oxygenation, melanin pigmentation, and density of macular pigment. Such quantitative analysis is often carried out through the use of a model of light propagation which attempts to explain the shape of remitted spectra by considering absorption and scattering by the structures and pigments present in the fundus. The general idea is that, given a set of physiological parameters $\mathbf{p}$ $=\left[\mathrm{p}_{1}, \ldots, \mathrm{p}_{\mathrm{k}}\right]$, a model is constructed that predicts the spectral reflectance $\mathrm{R}(\lambda, \mathbf{p})$. The image data at spatial location $\mathbf{x}$ can be computed as $I(\mathbf{x}, \lambda)=\int R[\lambda, \mathbf{p}(\mathbf{x})] \cdot S_{0}(\mathbf{x}, \lambda) \cdot Q(\lambda)$ $\cdot F(\lambda) \cdot d \lambda$ by removing the effects of the illuminant $\mathrm{S}_{0}(\mathbf{x}, \lambda)$, system optics $Q(\lambda)$, and any filters $F(\lambda)$. From these image data, the parameter vector $\mathbf{p}(\mathbf{x})$ that best describes the tissue at that point can be computed in a process known as "model inversion., ${ }^{5,11}$ Model inversion methods in multispectral imaging are similar to those used for data from single-point reflectometers, but the advantage of multispectral imaging is that it is possible to construct spatial maps showing the spatial distribution of chromophores across the fundus.

For the inversion methods to work it is essential that spectral information is represented accurately in a multispectral image set $\mathrm{I}(\mathbf{x}, \lambda)$. This imposes three requirements. First is that the image data are spatially aligned in the image stack, i.e., pixels $\mathrm{I}\left(\mathbf{x}, \lambda_{\mathrm{m}}\right)$ and $\mathrm{I}\left(\mathbf{x}, \lambda_{\mathrm{n}}\right)$ represent the same location in the fundus. Second, the spatial distribution of the illuminant must be independent of wavelength. The third is that the spectrum at every pixel contains sufficient information to recover the model parameters. The spectral resolution required for quantitative analysis varies strongly with wavelength, depending on the absorption characteristics of the chromophores of interest. For instance the region 540-600 $\mathrm{nm}$ is important for characterization of blood oxygenation and needs to have higher spectral resolution than the region beyond $620 \mathrm{~nm}$, where the spectra of the chromophores are relatively featureless.

There is no commercial multispectral retinal imaging system available, and a variety of different approaches have been used. The first class of system uses interferometry to acquire multispectral data. Spectral data are recovered from interferograms collected at each pixel location by Fourier transform. Whereas spectral resolution can be defined, it is not possible to acquire images at individually selected wavelengths or wavebands. ${ }^{12}$ These systems are popular in microscopy and remote sensing, but tend to have long acquisition times which make them fundamentally unsuitable for fundus imaging.

The second type of system in common use is the linescan or "push-broom" system, which acquires a spectrum from a single line of the image in a snapshot by dispersing the light from the line across the other dimension of the detector [typically a charge coupled device (CCD)]. The lines of the image are then scanned in turn to build up the multispectral image. Such systems have found application in fundus imaging, ${ }^{7}$ but share the same disadvantages as the interferometric systems, namely, slow imaging times [for a full two-dimensional (2D) image] and lack of wavelength selectivity at the time of acquisition.

Systems capable of the acquisition of images at specific wavelengths fall into three broad categories: (i) liquid crystal tunable filters ${ }^{5,8}$ that acquire images sequentially; (ii) a combination of dichroic mirrors and bandpass filters to simultaneously record multiple wavelengths on a single camera; ${ }^{9}$ and (iii) "snapshot" spectral imaging techniques that use holographic diffraction gratings to image multiple wavebands onto a single $\mathrm{CCD} .{ }^{8,10}$ Of these techniques, (i) has proven to be too slow when multiple wavelengths are required, ${ }^{5}$ and is rather expensive for use in routine clinical practice, while (ii) and (iii) do satisfy the requirement for fast acquisition speed and the ability to select wavelengths. However, since they record multiple wavebands on a single $\mathrm{CCD}$, there is necessarily a reduction in either image resolution or field of view.

It is clear that fundus imaging imposes a number of mutually conflicting constraints: short acquisition time, sufficiently high spatial and spectral resolution, and relatively low light levels to satisfy safety guidelines. ${ }^{13}$ The acquisition time problem cannot be overcome in either the Fourier domain or in the line-scan systems, but in sequential systems it can be reduced by imaging at selected wavelengths rather than uniformly across the whole spectrum. However, even with a reduced number of spectral frames, the acquisition time of a complete set of images could be greater that the natural timescale of the microsaccadic movements of the eye. As microsaccades occur typically at frequency of $\sim 1.5 \mathrm{~Hz}$, amplitude of $\sim 40$ arc min, ${ }^{14}$ this can result in significant movement between frames in the image stack. This violates the requirement of having spatial alignment between the frames. While this can be accurately corrected by image registration, the requirement that the spatial distribution of the illuminant should be independent of wavelength may still not be satisfied. The curvature of the fundus means that the region of interest is not evenly illuminated. Any movement of the fundus between the frames will result in a change of angle of illumination, and hence the incident light intensity, at a given spatial location. With different, and unpredictable, light levels it is impossible to ensure the correctness of spectral information which is necessary for model inversion.

Taking into account the above considerations we have designed a fast switching light emitting diode (LED) based discrete wavelength light source that can be used in combination with a high sensitivity CCD camera to obtain near movement free multispectral image sequences of the fundus. The system is capable of acquiring images at least one order of magnitude more quickly than the existing tunable filter system used previously by our group. ${ }^{5}$ We present our first images to be acquired with the new system, and analysis to show their suitability for the computation of chromophore distribution maps. 


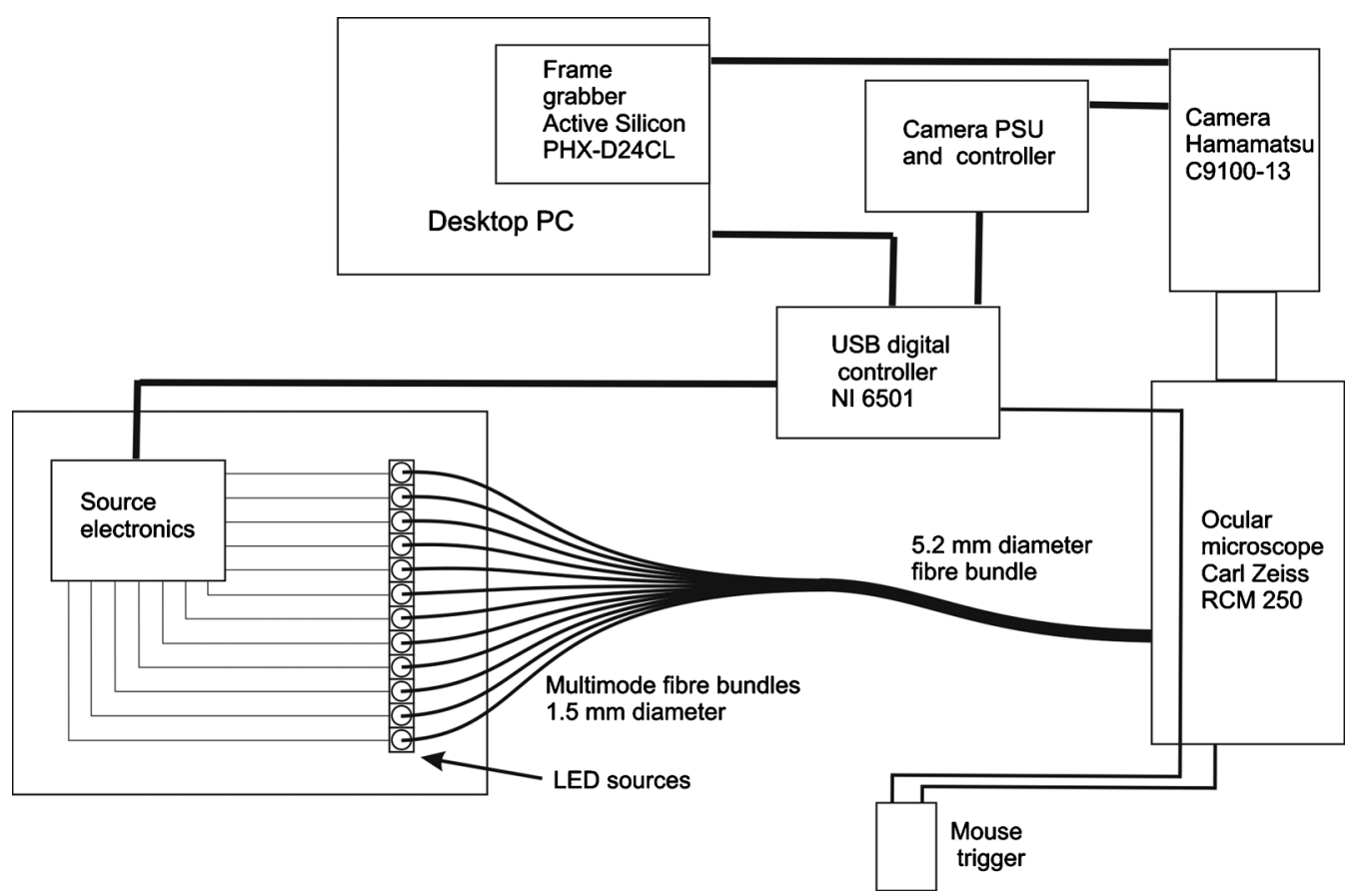

FIG. 1. Block diagram of retinal imaging system.

\section{THE SYSTEM}

\section{A. System overview}

The system consists of a fundus camera (Carl Zeiss RCM250), a bespoke LED based light source, a backilluminated electron multiplying CCD camera (Hamamatsu C9100-13) controlled by a frame grabber (Active Silicon PHX-D24CL) installed into a standard PC. The system is controlled by a National Instruments (Newbury, UK) USB digital controller (type 6501) that is controlled, in turn, by custom software developed with LABWINDOws (also National Instruments). This software ensures synchronization between the camera and the light source, so that image acquisition starts and stops precisely in time with each individual light pulse. The camera is controlled by a separate application, written in Visual $\mathrm{C}++$. A block diagram of the system is shown in Fig. 1.

\section{B. Background to light source design}

\section{System specification}

Source wavelengths. The design of the system is driven by the intended use of multispectral image data, that is, the derivation of quantitative maps of physiological parameters characterizing the retina. In an earlier study ${ }^{5}$ we have developed a multilayer model of the ocular fundus and a related inversion method. The layers [lens, intraocular medium, neural retina, retinal pigment epithelium (RPE), choroid, sclera] are characterized by their relevant generic optical parameters including wavelength-dependent absorption and scatter coefficients, refractive index, thickness, and concentration of chromophores. The chromophores to be recovered by inversion are retinal hemoglobins, choroidal hemoglobins, RPE melanin, and macular pigment. From the forward model of fundus reflectance we determined a set of optimal wavelengths $\boldsymbol{\lambda}_{0}$ based on two criteria: that there is one-to-one relationship between the image values and the parameters [i.e., the mapping between $\mathrm{I}\left(, \boldsymbol{\lambda}_{0}\right)$ and $\mathbf{p}$ is unique]; and that the wavelengths $\boldsymbol{\lambda}_{0}$ minimize the error of the parameter recovery process. This analysis suggested the following six wavelengths: 507, 525, 553, 584, 596, and $617 \mathrm{~nm}$ which were shown theoretically to separate the contributions of each chromophore. Further details can be found in Styles et $a l^{5}$ These wavelengths cover a wide range of the visible spectrum, from cyan through to red. Given how closely spaced some of these wavelengths are, it is essential that the bandwidth of each source is low-ideally less than $10 \mathrm{~nm}$.

Source luminance. It is necessary to deliver sufficient luminance at the retina to allow for short exposure times and therefore fast imaging. This is made harder by the low reflectivity of the fundus, especially at shorter visible wavelengths. There are also significant losses in the optics of the fundus camera.

Switching speed. It is essential to be able to switch rapidly between wavelengths, with minimal dead time between sources. The use of LEDs should allow exposure times of 10 $\mathrm{ms}$ or better to be achieved. However, based on the speed of the natural eye movements (saccades), an exposure time of less than 670 ms per multispectral image set should be sufficient as it would reduce the probability that a saccade would occur during the image acquisition process to a level that should produce acceptable images in the majority of cases.

Delivery via fiber optic bundle. Because of the very limited space available inside the fundus camera, the easiest way to deliver the light is via an optical fiber bundle.

\section{Possible designs for the light source}

We identified three possible approaches for delivering a system which can achieve the above requirements. 
White light source and wavelength selection. A combination of a tungsten filament source and an appropriate filter or monochromator is an obvious possibility. They are cheap, powerful, and have a relatively smooth spectrum. Flash lamps (i.e., discharge tubes) are also used in white light retinal imaging, but they have a major disadvantage for this application in that their spectrum is not smooth, making accurate quantitative numerical modeling of the imaging system and analysis of the images more difficult. The spectral distribution of the emitted power can also vary significantly from one discharge to the next. Another powerful source that could be considered is the supercontinuum laser. However, the main disadvantage of this would be its high cost.

An obvious possibility for wavelength selection is an acousto-optical filter - this has the advantage of very rapid switching between wavelengths but the disadvantage of being expensive. Other less expensive methods include chromatic dispersion (e.g., by using a prism or grating) or the use of narrowband filters. Interference filters would be the most appropriate choice here in order to produce a sufficiently narrow wavelength range. All these methods have the disadvantage of requiring a mechanical system to change wavelength. In the case of a prism or a diffraction grating, either they would have to be rotated, or a fiber bundle would have to be scanned across the emitted spectrum. In the case of interference filters, a device such as a filter wheel would be necessary, so that each filter could be moved rapidly in front of the light source in turn. However it is fairly straightforward to transmit sufficient light through such a filter, and the transmission ratio between the pass band and the stop band is excellent.

Tunable source. Optical parametric oscillator based lasers could be considered as a source. However, they have two major disadvantages: they are expensive, and the tuning is performed mechanically and is therefore slow.

Discrete sources at different wavelengths. A third alternative is to use discrete sources, such as laser diodes or high power LEDs. Laser diodes have the advantage of producing light of a very narrow bandwidth (typically less than $1 \mathrm{~nm}$ ). Unfortunately the range of wavelengths available in the visible region of the spectrum is severely limited. Conversely, LEDs have a much higher range of available wavelengths, although with the disadvantage of having a broader emission bandwidth - typically between 20 and $30 \mathrm{~nm}$. In the past few years high power LEDs have become cheap and commonplace-for a few pounds a single device emitting typically $40 \mathrm{~lm}$ can be obtained. This plentiful source power makes narrowband filtering much easier, as the inevitable transmission loss does not have to be a major concern. Using interference filters a $20-30 \mathrm{~nm}$ bandwidth can easily be reduced to $5-10 \mathrm{~nm}$.

From all these possibilities, two obvious contenders emerge:

(1) a tungsten light source with a filter wheel; and

(2) discrete LED sources with filters.

Option 1 has much to recommend it, in particular its simplicity. However, a system involving moving mechanical parts can sometimes require a disproportionate effort in de- sign and construction of these parts. For this reason, if a system can be designed to be "solid state," this is often preferable. Also, a discrete LED design allows much better control of the relative power outputs of the sources. These were the overriding advantages which led us to develop a system based on option 2 .

\section{Light source design}

\section{Summary}

The light source we have developed for the fundus camera is based on a previous design that was employed in a near-infrared optical topography system. ${ }^{15}$ The source consists of up to 12 LEDs that can be programmed to illuminate in any way that the user chooses, limited only by the speed of the digital electronics. It has a modular design that allows LEDs of different wavelengths to be easily inserted into the system for use in different applications. Each LED is individually addressable, allowing precise control of intensity, illumination time, and illumination sequence. The source is coupled to the fundus camera using a 12 way fiber optic bundle (described below). This is inserted into the camera to sit at the focus of the collimating lens of the xenon light source that has been removed.

\section{Design in detail}

Choice of LEDs. High output power was a major priority for this application, so we identified the most powerful LEDs that were commercially available as a single device. At the time this was the Philips "Lumileds Luxeon Star" range (San Jose, U.S.A.). These dissipate roughly $1 \mathrm{~W}$ of electrical power and have a typical luminous flux of $\sim 40 \mathrm{~lm}$. They are fitted with a collimating optic which results in a typical on-axis luminous intensity of $600 \mathrm{~cd}$. At maximum power these LEDs pass a current of $350 \mathrm{~mA}$. We used devices of five different wavelengths: 505, 530, 557, 590 , and $617 \mathrm{~nm}$, chosen to match the six interference filter wavelengths as closely as possible.

Choice of filters. LEDs typically have a bandwidth of 20-30 nm. However, for this retinal imaging application, a narrower bandwidth is required. This can be achieved using interference filters, which have around $90 \%$ transmission in their pass band with a full width at half maximum value of around $12 \mathrm{~nm}$. They also have very good attenuation characteristics in the stop band, with typical optical densities here no worse than 6 . We were able to select filters for all but one wavelength from the Semrock Brightline (R) single bandpass range (Rochester, New York, USA). There was no suitable filter in this range for the target wavelength of $553 \mathrm{~nm}$. We obtained this filter from Spectrogon (Taby, Sweden) from their narrow bandpass filter range. Only one of the filters $(525 \mathrm{~nm})$ could be exactly matched to the desired wavelength as determined by the previous analysis. ${ }^{5}$ The other five are all slightly different. This is simply due to the limited range of filter wavelengths available commercially. The mismatches are only a few nanometers (at most $4 \mathrm{~nm}$ ). It was determined that this would not significantly affect the chromophore analysis algorithm. ${ }^{5}$ 
Optical fiber bundle design. Given the limited space available inside the fundus camera, it was decided that a fiber bundle was the best way of delivering the light to it. A 1.5 $\mathrm{mm}$ diameter fiber bundle is attached to each LED/filter unit. The fibers from these 12 bundles are then gathered together incoherently into one larger bundle, so that each source wavelength is distributed as evenly as possible across the end of the bundle. This is approximately $5 \mathrm{~mm}$ in diameter. The optical fiber used is Schott type B3 (Southbridge, Massachusetts, USA), which has a diameter of $30 \mu \mathrm{m}$ and a numerical aperture (NA) of 0.54 . The core is $25 \mu \mathrm{m}$ in diameter so the core to cladding ratio is high. This thin cladding maximizes the packing fraction of the bundle, thereby increasing the efficiency of light transmission through the bundle. The fiber material is glass, which has excellent transmission characteristics throughout the visible spectrum. The total length of the fiber bundle is $1 \mathrm{~m}$, with the section where the 12 bundles are gathered together being $80 \mathrm{~cm}$ long.

Arrangement of LEDs and filters-coupling to optical fiber bundle. The attached optics collimate the light being emitted from the LEDs. This is important as the interference filters have a maximum acceptance half angle of $7^{\circ}$. Ideally, a further converging lens should be placed in the light path to maximize the amount of light that is coupled into the fiber bundle. However, for simplicity in this prototype system, this was not done. The fiber bundle end was simply placed in contact with the surface of the interference filter. The optic collimates the LED light to a diverging beam with an $\sim 10^{\circ}$ half angle. Knowing this and the on-axis luminous intensity (640 $\mathrm{lm} / \mathrm{sr}$ for the $605 \mathrm{~nm}$ source) allows a crude estimation of the coupling efficiency of the arrangement. Ignoring the attenuation due to the filter, approximately $1 \%$ of the total output of the LED is coupled into the fiber bundle. Power measurements on a typical source (the $605 \mathrm{~nm}$ one) show that the power delivered at the far end of the optical fiber bundle is approximately $2 \%$ of the total output from the LED - and this includes the loss due to the interference filter. While this is not the most efficient arrangement possible, it was found to produce a sufficient light output for the retinal imaging application.

Design of system electronics. The existing topography system electronics ${ }^{15}$ were adapted to drive the high power LEDs, which can draw currents of up to $350 \mathrm{~mA}$ - a simple metal-oxide semiconductor field-effect transistor (MOSFET) common source amplifier was added to the system. Each LED can be controlled by the PC, from fully off to maximum power in 100 incremental steps. The system is controlled by a simple USB digital input/output device (National Instruments 6501). It is powered by a switched mode medical grade power supply and housed in a standard 19 in. subrack and case.

\section{New CCD imager for fundus camera}

The Zeiss fundus camera used for this project dates from approximately 20 years ago. We chose to use this older system as it was much easier to modify for our purposes than a modern one would have been-this is because of the more integrated and complex electronics used in modern fundus cameras. The original SLR camera was replaced with a

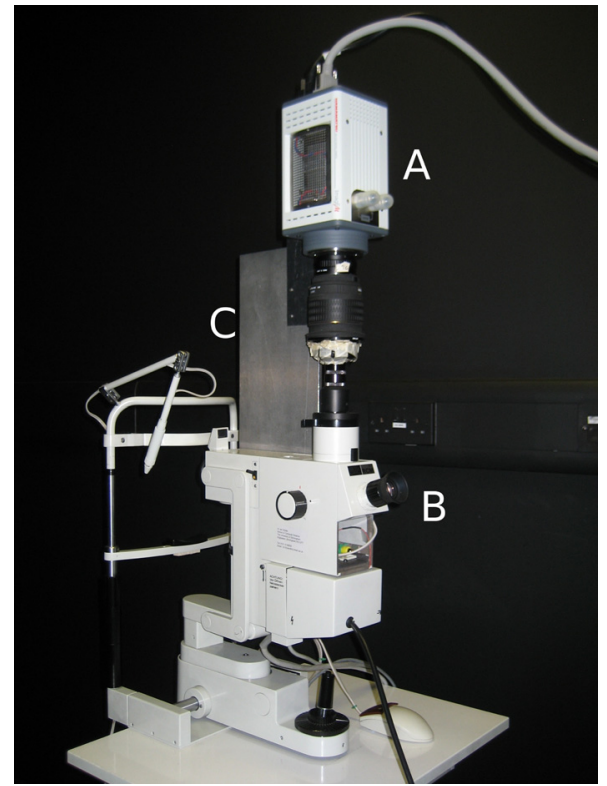

FIG. 2. (Color online) CCD imager attached to fundus camera. A-CCD camera, B-viewing eyepiece, $\mathrm{C}$ - support structure for camera.

Hamamatsu C9100-13 video camera, a back-thinned and electron multiplying CCD based device, with a 512 $\times 512$ pixel sensor, and 16 bits of digitization. This is a very sensitive camera designed to be able to do fast imaging at very low light levels. It is easily capable of recording at the frame rates required for this system (maximum 400/s), and the images it obtains in this application are photon-noise limited. It was attached to the fundus camera with a suitable macrolens (Sigma Aspherical $24 \mathrm{~mm}$ F1.8 Macro) to ensure the correct optical coupling between the fundus camera and the imaging head. This lens moves the image plane from the position appropriate to the SLR camera body to the new position required by the $\mathrm{CCD}$ camera. It also resizes the image to fit onto the smaller CCD imaging area. This is a square with sides of $8.19 \mathrm{~mm}$, considerably smaller than the image area used in a $35 \mathrm{~mm}$ camera, which is typically 36 $\times 24 \mathrm{~mm}$. The camera head weighs $3.7 \mathrm{~kg}$ so it was necessary to mount it on a support structure attached to a loadbearing part of the fundus camera (see Fig. 2).

\section{E. Other modifications to the fundus camera}

The normal triggering mechanism of the fundus camera has been replaced with a three button system. The buttons can be used individually during the system development and testing, or together during the image acquisition session, using a switch plate that overlays the three buttons. One button serves as the trigger which initiates the imaging sequence. This button is connected to the USB I/O device, which then coordinates the timing of all the system components. The other two buttons allow individual manual control of the fundus camera mirrors. These mirrors rotate under the control of electromagnetic solenoids. The first switches the light path between the viewing eyepiece and the camera, and the second changes the illumination source between the tungsten filament lamp (used for focusing) and the LED light source (used for imaging). This mirror was found to provide insuf- 
ficient optical isolation from the tungsten filament lamp during image acquisition. For this reason a relay circuit was added to the fundus camera, so that the filament lamp could be switched off during the imaging sequence.

The optical fiber bundle was inserted into the system so that the end was located at the point formerly occupied by the xenon flash lamp, which had been removed. In this way the new source can employ the same optics, and therefore have the same light path through the instrument. The Maxwellian view optics of the fundus camera redistribute the fiber output to give annular illumination at the pupil, with imaging performed through the center of the annulus.

\section{F. System integration, control, and safety}

The entire system, including the PC, is run from a medical grade isolation transformer. A block diagram of the system is shown in Fig. 1, illustrating the interconnections of the various components. All connections are via the digital controller (USB 6501) which coordinates the actions of each component.

The system is run from a standard desktop PC running WINDOWS XP. The light source is controlled by an application written in the LABWINDOWS development environment (National Instruments, Newbury, U.K.). A graphical user interface controls the system. The intensity and duration of each source pulse can be set independently, and sources can be included or excluded from the pulse sequence as needed. The order in which the sources pulse can also be changed. The number of repetitions of the pulse sequence can be set, or the system can be set to run continuously. The pulse sequence can be started from the user interface or from the trigger button attached to the fundus camera.

It is of paramount importance that the system does not expose the eye to unsafe light levels. The maximum possible exposure from each source at the retina has been measured and compared with the relevant safety limits, with particular reference to the criteria for a Maxwellian viewing geometry with a $45^{\circ}$ viewing angle defined by Sliney et al. ${ }^{16}$ We found that the brightest source, at $2.5 \mathrm{~mW}$, was a factor of 20 below the stated safety limit (of $50 \mathrm{~mW}$ entering the eye). This renders the system safe in normal use. If the control electronics were to fail and all the sources were to be illuminated simultaneously, the system would still be eye safe by a factor of 13 .

\section{G. Final system specification}

The current version of the system has six narrowband sources with wavelengths of 504, 525, 557, 578, 605, and $620 \mathrm{~nm}$. The optical power they can deliver to the far end of the fiber bundle was measured and found to be 1.7, 0.6, 0.6, $0.2,1.2$, and $2.9 \mathrm{~mW}$, respectively. These variations in power output are largely due to the unavoidable mismatches between the center wavelength of emission from the LED and the center wavelength of the respective filter. The LEDs have very fast rise and fall times - they can be switched on and off in less than $100 \mathrm{~ns}$.
TABLE I. Image acquisition parameters used for retinal imaging: central wavelength of the filtered LED, the source intensity, and the exposure duration.

\begin{tabular}{ccc}
\hline \hline $\begin{array}{c}\text { Wavelength } \\
(\mathrm{nm})\end{array}$ & $\begin{array}{c}\text { Intensity } \\
(\mathrm{mW})\end{array}$ & $\begin{array}{c}\text { Duration } \\
(\mathrm{s})\end{array}$ \\
\hline 504 & 0.47 & 0.05 \\
525 & 0.45 & 0.05 \\
557 & 0.05 & 0.20 \\
582 & 0.15 & 0.05 \\
600 & 0.21 & 0.05 \\
620 & 1.55 & 0.05 \\
\hline \hline
\end{tabular}

\section{H. System operation}

The image capturing procedure is as follows. The light source parameters are set using the graphical user interface. The CCD imaging parameters are set similarly using its user interface. The operator adjusts the fundus camera using the existing tungsten filament illumination to obtain the best image of the fundus of the eye. The three control buttons are then depressed simultaneously. This actuates the two mirrors of the fundus camera-one to switch the light source from tungsten to LED, the other to switch the viewing path from the operator's eyepiece to the CCD camera. At the same time the third button sends a digital trigger to the PC that starts the light source sequence and the camera imaging sequence simultaneously. The digital controller switches on each LED in the sequence in turn, illuminating the retina. Directly after each LED is turned on a synchronization pulse is sent from the USB controller to the camera controller to start a frame exposure. This same pulse finishes the previous exposure. The LED is then switched off, and the next LED is illuminated-immediately followed by the next synchronization pulse. In this way it is assured that one wavelength of illumination cannot "bleed" into the next exposure. With this method many short exposure images of the fundus can be obtained consecutively, limited only by subject tolerance to the light source. The PC emits a sound to notify the operator when the exposure sequence is complete.

\section{TESTING AND PRELIMINARY RESULTS}

\section{A. Testing protocol}

The performance of the imager was evaluated to assess whether or not the multispectral image sets it produces are impaired by the microsaccadic motion of the eye (see Sec. I). At $1.5 \mathrm{~Hz}$ the typical duration of the intersaccadic interval is $0.67 \mathrm{~s}$. Therefore if images at the six selected wavelengths (see Sec. II B) are acquired within this time, the chance of eye movement between the frames is minimized. Moreover, if three sets of six images are taken sequentially, at least one of the sets is likely to capture the image data between the saccades.

These considerations led to the following imaging protocol. After a satisfactory focus is obtained three contiguous sets of exposures are obtained at the wavelengths shown in Table I. The images are acquired in order of increasing wavelength, and three complete sequences of images (18 images in total) are acquired. The table shows the intensity and du- 

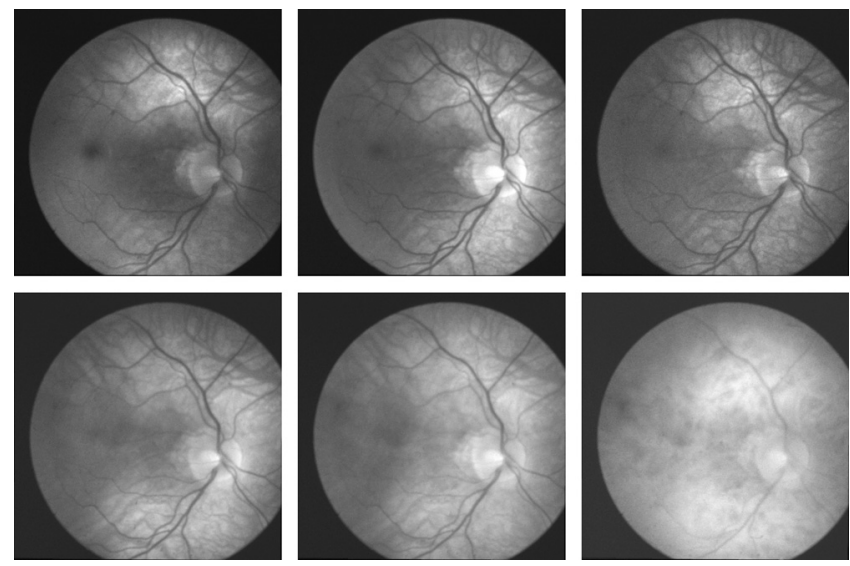

FIG. 3. Images from subject B-from left to right and top to bottom, the wavelengths are 504, 525, 557, 582, 600, and $620 \mathrm{~nm}$. The images shown are an average of three exposures and have been scaled for display purposes to the same minimum and maximum intensity and gamma corrected with a coefficient of 0.7 . The reduction in visible features as the wavelength increases can be clearly seen. This is caused by the lower absorption coefficient of both hemoglobins and melanin at the longer wavelengths.

ration of illumination at each wavelength, both established through preliminary experimentation. The camera electronmultiplying gain is set to the same value of 400 for all the exposures. The longer exposure for the $557 \mathrm{~nm}$ source was due to its weaker output power, a problem for which a solution has been identified.

\section{B. Evaluation method}

One of the key requirements of the new system was that the images acquired at the six consecutive wavelengths should show minimal displacement. The displacement was estimated from the parameters of the transformation matrices returned by the image registration algorithm of Stewart et $a l .{ }^{17}$ This algorithm was developed specifically for the alignment and mosaicing of fundus images. Images are registered pair-wise, and for each pair registered the algorithm returns parameters of the biquadratic warping function which account for translation, rotation, and peripheral warping,

$$
\begin{aligned}
& x^{\prime}=a_{0}+a_{1} x+a_{2} y+a_{3} x^{2}+a_{4} y^{2}+a_{5} x y, \\
& y^{\prime}=b_{0}+b_{1} x+b_{2} y+b_{3} x^{2}+b_{4} y^{2}+b_{5} x y,
\end{aligned}
$$

where $\left[\begin{array}{l}a_{0} \\ b_{0}\end{array}\right]$ defines translation, $\left[\begin{array}{ll}a_{1} & b_{1} \\ a_{2} & b_{2}\end{array}\right]$ defines rotation, and $\left[\begin{array}{lll}a_{3} & a_{4} & a_{5} \\ b_{3} & b_{4} & b_{5}\end{array}\right]$ defines warp. As preliminary experiments on human subjects clearly showed that rotation and warping are negligible, only the translation parameters were used in the evaluation. Image registration was carried out in two ways: between the consecutive images within the set (i.e., image at $504 \mathrm{~nm}$ to image at $525 \mathrm{~nm}$, image at $525 \mathrm{~nm}$ to image at $557 \mathrm{~nm}$, etc.); and between the second image in the set (i.e., at $525 \mathrm{~nm}$ ) and all the remaining images in that set. The results of registration were checked visually. Automatic registration was unreliable for most of images at $620 \mathrm{~nm}$; as can be seen from the typical example in Fig. 3, at this wavelength hardly any vascular features are present. For this reason these frames were excluded from the evaluation.
TABLE II. Linear displacement $d=\sqrt{a_{0}^{2}+b_{0}^{2}}$ between two image frames taken at different wavelengths of the static artificial eye. Only the results of the sequential registration are shown as the results of registration to the image at $525 \mathrm{~nm}$ were very similar. Values are shown in pixels (pixel size is approximately $20 \mu \mathrm{m})$.

\begin{tabular}{lcccc}
\hline \hline & $\min d$ & $\max d$ & Mean & st.dev \\
\hline Artificial eye & 0.01 & 2.30 & 0.08 & 0.90 \\
\hline \hline
\end{tabular}

\section{Testing to establish a registration baseline}

The system was first tested using a phantom that mimics the appearance and optical properties of the real human eye; henceforth referred to as an artificial eye. In order to establish a baseline against which the retinal image data could be compared, nine multispectral image sets of the artificial eye placed at different orientations with respect to the imaging plane were taken. Images forming each multispectral set were registered using both regimes (sequential and each to the $525 \mathrm{~nm}$ image), as described in Sec. III B above. Since there was no movement of the artificial eye between the frames, the expected value of the translation vector $d$ $=\sqrt{a_{0}^{2}+b_{0}^{2}}$ returned by the registration algorithm was $d=0$. Table II summarizes the actual results.

The observed small displacement can be attributed to a combination of errors due to image noise, and possibly to minute vibrational movements caused by the camera's cooling system. The former arises from the fact that the image registration algorithm uses vascular features such as junctions and centerlines of the retinal vessels to align a pair of images. Noise can cause a small error in the location of the detected features. The maximum observed displacement of 2.3 pixels was taken to be the system error and used as a benchmark for the subsequent experiments.

\section{Testing on retinal images}

Image sets were acquired from two volunteers using the protocol described in Sec. III A. Subject A was a Caucasian female and subject B was a Caucasian male. In Fig. 3, we show images from subject B.

The registration analysis, as described above for the artificial eye, was performed on the image sets. Table III shows the linear displacement $d$ for each of the three sixwavelength sequences, for both the sequential registration and registration to an image at $525 \mathrm{~nm}$.

We have also analyzed the quality of the images by computing their signal to noise ratio (SNR). This was done from two regions of the image: the macula and a region of the retina away from major vessels. The results of this analysis are shown in Table IV.

\section{DISCUSSION AND CONCLUSIONS}

\section{A. Performance}

The experiments have demonstrated that a multispectral image data set of a satisfactory quality can be acquired in less than $0.5 \mathrm{~s}$, thus meeting a theoretically predicted threshold of $0.67 \mathrm{~s}$ (see Sec. III A). The expectation was that one in three multispectral sequences would be free of microsaccadic 
TABLE III. Linear displacement $d=\sqrt{a_{0}^{2}+} b_{0}^{2}$ showing the magnitude of translation required to align two images taken at five different wavelengths in the same sequence. $\left[a_{0} b_{0}\right]^{\mathrm{T}}$ is a translation vector returned by the registration algorithm (Ref. 17) (see Sec. III B). Values are shown in pixels (pixel size is approximately $20 \mu \mathrm{m})$.

\begin{tabular}{|c|c|c|c|c|c|c|c|c|}
\hline & \multicolumn{4}{|c|}{ Sequential registration } & \multicolumn{4}{|c|}{ Registration to $525 \mathrm{~nm}$ image } \\
\hline & $\min d$ & $\max d$ & Mean & st. dev & $\min d$ & $\max d$ & Mean & st. dev \\
\hline \multicolumn{9}{|l|}{ Subject A } \\
\hline Sequence 1 & 0.75 & 2.30 & 1.52 & 0.70 & 0.64 & 1.85 & 1.03 & 0.56 \\
\hline Sequence 2 & 0.37 & 1.49 & 0.92 & 0.46 & 0.37 & 2.30 & 1.06 & 0.85 \\
\hline Sequence 3 & 0.72 & 2.21 & 1.29 & 0.67 & 0.68 & 1.78 & 1.67 & 0.48 \\
\hline \multicolumn{9}{|l|}{ Subject B } \\
\hline Sequence 1 & 0.20 & 1.64 & 1.11 & 0.66 & 0.39 & 1.53 & 0.95 & 0.48 \\
\hline Sequence 2 & 0.48 & 1.36 & 0.97 & 0.41 & 0.63 & 1.53 & 1.19 & 0.39 \\
\hline Sequence 3 & 0.98 & 2.17 & 1.36 & 0.55 & 0.35 & 1.72 & 1.03 & 0.56 \\
\hline
\end{tabular}

TABLE IV. SNR (decibel) measurements from subject B. A $64 \times 64$ pixel region was used from each of the respective areas. The SNR was computed using SNR $=20 \log _{10}\left(\mathrm{rms} \_\right.$signal/rms_noise $)$.

\begin{tabular}{lcccccc}
\hline \hline & & & & & & \\
Wavelength $(\mathrm{nm})$ & 504 & 525 & 557 & 582 & 600 & 620 \\
Macular region & 14.2 & 13.9 & 11.1 & 14.1 & 14.8 & 16.7 \\
Normal retina & 16.7 & 25.1 & 15.4 & 23.2 & 23.2 & 20.8 \\
\hline \hline
\end{tabular}

movement. Interestingly, none of the in vivo sequences show a within-the-set displacement of greater than 2.3 pixels, which is the same as that observed in images of the static artificial eye. With a pixel size of the order of $20 \mu \mathrm{m}$ such small displacements ensure near-equal illumination for all the multispectral frames and provide good alignment for all retinal features, perhaps with the exception of small capillaries. Light levels used for illumination with the LED sources are within the safety limit recommendations of the American National Standards Institute ${ }^{13}$ (approximately $1 \mathrm{~mJ} / \mathrm{cm}^{2}$, which is under $1 \%$ of the maximum permissible exposure).

\section{B. Conclusions}

We have demonstrated that LED illumination combined with a high-sensitivity CCD camera is capable of acquiring high quality multispectral images of the human fundus. In this work we have employed a particular set of wavelengths that our research found to be suitable for quantifying chromophores present in the fundus of the eye. However, the flexible nature of the light source means that wavelengths can be easily changed.

We have identified the following advantages of our system compared to other multispectral retinal imaging systems. (i) The system can acquire images at a high enough rate to reduce the occurrence of motion artifacts in the multispectral image cube to an acceptable level. (ii) the spatial resolution and field of view are not compromised as they are in the CCD-based snapshot systems. (iii) The system uses only the specific wavebands that are required for the subsequent analysis, minimizing the total light exposure of the subject (interferometric and snapshot systems require a white-light source). (iv) The modular nature of the light source allows new wavelengths to be introduced very easily, and at very low cost.
We have also identified several potential disadvantages. In particular, we note that it is not always possible to obtain LEDs at the desired wavelengths; and that while the experiments described here have demonstrated that the system is fast enough to acquire images without significant interframe motion, it does not capture a snapshot image. This would be possible, but would either require loss of resolution or field of view, or would require multiple cameras which would be costly. We therefore conclude that our system is suitable for quantitative imaging of the fundus, as well as being economically viable.

We aim to investigate the use of the new system in conjunction with the image analysis algorithms already developed $^{5}$ to image and characterize a variety of ocular diseases with a particular focus on AMD, where our approach has already shown significant promise. ${ }^{5}$ The long term goal is to develop a system for chromophore quantification that is an integral part of the standard fundus camera, and therefore could be used routinely by both optometrists and ophthalmologists.

\section{ACKNOWLEDGMENTS}

This research was funded by the Engineering and Physical Sciences Research Council (EPSRC). Thanks also go to Joe Evans, Erich Herrmann, and Rob Cooper for their valuable help.

${ }^{1}$ J. A. Olson, F. M. Strachan, J. H. Hipwell, K. A. Goatman, K. C. McHardy, J. V. Forrester, and P. F. Sharp, Diabetic Med. 20, 528 (2003).

${ }^{2}$ C. A. Puliafito, M. R. Hee, C. P. Lin, E. Reichel, J. S. Schuman, J. S. Duker, J. A. Izatt, E. A. Swanson, and J. G. Fujimoto, Ophthalmology 102, 217 (1995)

${ }^{3}$ M. J. Greaney, D. C. Hoffman, D. F. Garway-Heath, M. Nakla, A. L. Coleman, and J. Caprioli, Invest. Ophthalmol. Visual Sci. 43, 140 (2002). 
${ }^{4}$ A. M. Zysk, F. T. Nguyen, A. L. Oldenburg, D. L. Marks, and S. A. Boppart, J. Biomed. Opt. 12, 051403 (2007).

${ }^{5}$ I. B. Styles, A. Calcagni, E. Claridge, F. Orihuela-Espina, and J. M. Gibson, Med. Image Anal. 10, 578 (2006).

${ }^{6}$ A. R. Harvey, J. Lawlor, A. I. McNaught, J. W. Williams, and D. W. Fletcher-Holmes, Proc. SPIE 4816, 325 (2002).

${ }^{7}$ D. Schweitzer, M. Hammer, and M. Scibor, Ophthalmic Res. 28, 37 (1996).

${ }^{8}$ I. Alabbouda, G. Muyo, A. Gorman, D. Mordant, A. McNaught, C. Petres, Y. R. Petillot, and A. R. Harvey, Proc. SPIE 6631, 1 (2007).

${ }^{9}$ J. M. Beach, K. J. Schwenzer, S. Srinivas, D. Kim, and J. S. Tiedeman, J. Appl. Physiol. 86, 748 (1999).

${ }^{10}$ W. R. Johnson, D. W. Wilson, W. Fink, M. Humayun, and G. Bearman, J. Biomed. Opt. 12, 014036 (2007).

${ }^{11}$ T. T. J. M. Berendschot, P. J. DeLint, and D. van Norren, Prog. Retin Eye
Res. 22, 171 (2003).

${ }^{12}$ M. Lavi, U. Milman, D. Cabib, Y. Garini, A. Gil, T. Juta, and M. E. Adel, Proc. SPIE 3261, 313 (1998).

${ }^{13}$ F. C. Delori, R. H. Webb, and D. H. Sliney, J. Opt. Soc. Am. A 24, 1250 (2007).

${ }^{14} \mathrm{H}$. Arimoto and H. Furukawa, Engineering in Medicine and Biology Society, 2007 (EMBS 2007). 29th Annual International Conference of the IEEE, 22-26 August 2007, pp. 1627-1630.

${ }^{15}$ N. L. Everdell, A. P. Gibson, I. D. C. Tullis, T. Vaithianathan, J. C. Hebden, and D. T. Delpy, Rev. Sci. Instrum. 76, 093705 (2005).

${ }^{16}$ D. Sliney, D. Aron-Rosa, F. DeLori, F. Fankhauser, R. Landry, M. Mainster, J. Marshall, B. Rassow, B. Stuck, S. Trokel, T. Motz West, and M. Wolffe, Appl. Opt. 44, 2162 (2005).

${ }^{17}$ C. V. Stewart, C.-L. Tsai, and B. Roysam, IEEE Trans. Med. Imaging 22, 1379 (2003). 